\title{
Symmetry finder: A method for hunting symmetry in neutrino oscillation
}

\author{
Hisakazu Minakata๑* \\ Center for Neutrino Physics, Department of Physics, Virginia Tech, Blacksburg, Virginia 24061, USA
}

(Received 26 June 2021; accepted 27 September 2021; published 18 October 2021)

\begin{abstract}
Symmetry in neutrino oscillation serves for a better understanding of the physical properties of the phenomenon. We present a systematic way of finding symmetry in neutrino oscillation, which we call symmetry finder (SF). By extending the known framework in vacuum into a matter environment, we derive the SF equation, a powerful machinery for identifying symmetry in the system. After learning lessons on symmetry in the Zaglauer-Schwarzer system with matter equivalent to the vacuum symmetry, we apply the SF method to the [P. B. Denton et al., Compact perturbative expressions for neutrino oscillations in matter, J. High Energy Phys. 06 (2016) 051.] (DMP) perturbation theory to first order. We show that the method is so powerful that we uncover the eight reparametrization symmetries with the $1 \leftrightarrow 2$ state exchange in DMP, denoted as IA, IB, ..., IVB, all new except for IA. The transformations consist of both fundamental and dynamical variables, indicating their equal importance. It is also shown that all the symmetries discussed in this paper can be understood as the Hamiltonian symmetries, which ensures their all-order validity and applicability to varying density matter.
\end{abstract}

DOI: 10.1103/PhysRevD.104.075024

\section{INTRODUCTION}

In the theory of neutrino oscillation symmetry plays an important role, yielding useful relations between the observables. $C P T$ symmetry implies that the neutrino and antineutrino probabilities are equal, $P\left(\nu_{\beta} \rightarrow \nu_{\alpha}\right)=$ $P\left(\bar{\nu}_{\beta} \rightarrow \bar{\nu}_{\alpha}\right)$ in vacuum. Time reversal $T$ : Even though $T$ symmetry itself is broken in nature [1], it yields a generalized symmetry, an invariance of the $S$ matrix under $T$ transformation accompanied with complex conjugation of the complex numbers in the theory. The generalized $T$ symmetry holds not only in vacuum but also in matter. As symmetry often brings us useful consequences, finding symmetry and its use in the exploration of physical properties of neutrino oscillations should lead us to a deeper understanding of the phenomenon.

Not so surprisingly, $T$-odd quantities obey a few remarkable identities and general regularities in the oscillation probability. They include the Naumov [2] and Toshev [3] identities, whose former implies that the Jarlskog factor in matter must be proportional [4] to the Jarlskog factor in vacuum, $J_{r} \equiv c_{12} s_{12} c_{13}^{2} s_{13} c_{23} s_{23}$ [5]. The dependence of the $C P$ phase $\delta$, the lepton counterpart of the quark $C P$ phase [6], on the oscillation probability is also strongly

\footnotetext{
*hisakazu.minakata@gmail.com
}

Published by the American Physical Society under the terms of the Creative Commons Attribution 4.0 International license. Further distribution of this work must maintain attribution to the author(s) and the published article's title, journal citation, and DOI. Funded by SCOAP. constrained in matter to the single and double harmonics (sine and cosine of $\delta$ and $2 \delta$ ) [7], which allows simple and informative representations by the $C P$ - or $T$-conjugate biprobability diagrams $[8,9]$. For a broader view of $C P$ and $T$ violation see, e.g., Ref. [10].

It is the purpose of this paper to present a systematic way of uncovering symmetry in neutrino oscillations. Such a method should be welcome as it makes symmetry consideration handy and thereby contributes to a clearer physics understanding. Now, we must note that symmetry in neutrino oscillation is not necessarily the one which contains spacetime reflection, despite that we have started our description from it. In a perturbative treatment of neutrino oscillation by Denton et al. [11], dubbed as DMP hereafter, a relabeling or reparametrization symmetry is found. It is an invariance of the probability under the transformations $\lambda_{1} \leftrightarrow \lambda_{2}, \cos \psi \rightarrow \mp \sin \psi, \sin \psi \rightarrow \pm \cos \psi$. Here, $\lambda_{i} / 2 E \quad(i=1,2,3)$ denote the eigenvalues of the Hamiltonian with $E$ being neutrino energy and $\psi$ the mixing angle $\theta_{12}$ in matter. In this paper we will find a new family of these symmetries in the same theory.

The other examples of the reparametrization symmetry with the eigenstate exchange in the solar- and atmosphericresonance perturbation theories are later discussed in Ref. [12]. An interpretation of these symmetries as the "dynamical symmetry," as opposed to the Hamiltonian

\footnotetext{
${ }^{1}$ A dynamical symmetry is the symmetry that has no hint in the Hamiltonian of the system, but the one which indeed arises after the system is solved. The symmetry often comes with the transformations written by the variables that are used to diagonalize the Hamiltonian.
} 
TABLE I. All the symmetries of the $1 \leftrightarrow 2$ eigenstate exchange type in the DMP system that have been uncovered in this paper are summarized. They are found in a systematic way by solving the SF equation with appropriate ansatz, as some of them (symmetries IA, IB, and IVB) are fully explained in Sec. V. The symmetry denoted as, e.g., "symmetry X" in this table is called "symmetry X-DMP" in the text, where $\mathrm{X}=\mathrm{IA}$, IB, IIA, IIB, IIIA, IIIB, IVA, and IVB. In this table the notations are such that $\lambda_{j}(j=1,2)$ are the eigenvalues of $2 E H$, and $\psi$ and $\phi$ denote $\theta_{12}$ and $\theta_{13}$ in matter, respectively.

\begin{tabular}{|c|c|c|}
\hline Symmetry & Vacuum parameter transformations & Matter parameter transformations \\
\hline Symmetry IA & None & \multirow{8}{*}{$\begin{array}{c}\lambda_{1} \leftrightarrow \lambda_{2}, c_{\psi} \rightarrow \mp s_{\psi}, s_{\psi} \rightarrow \pm c_{\psi} . \\
\lambda_{1} \leftrightarrow \lambda_{2}, c_{\psi} \rightarrow \pm s_{\psi}, s_{\psi} \rightarrow \pm c_{\psi} . \\
\lambda_{1} \leftrightarrow \lambda_{2}, c_{\psi} \rightarrow \pm s_{\psi}, s_{\psi} \rightarrow \pm c_{\psi} . \\
\lambda_{1} \leftrightarrow \lambda_{2}, c_{\psi} \rightarrow \mp s_{\psi}, s_{\psi} \rightarrow \pm c_{\psi} . \\
\lambda_{1} \leftrightarrow \lambda_{2}, \phi \rightarrow-\phi, \\
c_{\psi} \rightarrow \pm s_{\psi}, s_{\psi} \rightarrow \pm c_{\psi} \\
\lambda_{1} \leftrightarrow \lambda_{2}, \phi \rightarrow-\phi, \\
c_{\psi} \rightarrow \mp s_{\psi}, s_{\psi} \rightarrow \pm c_{\psi} . \\
\lambda_{1} \leftrightarrow \lambda_{2}, \phi \rightarrow-\phi, \\
c_{\psi} \rightarrow \mp s_{\psi}, s_{\psi} \rightarrow \pm c_{\psi} . \\
\lambda_{1} \leftrightarrow \lambda_{2}, \phi \rightarrow-\phi, \\
c_{\psi} \rightarrow \pm s_{\psi}, s_{\psi} \rightarrow \pm c_{\psi} .\end{array}$} \\
\hline Symmetry IB & $\theta_{12} \rightarrow-\theta_{12}, \delta \rightarrow \delta+\pi$ & \\
\hline Symmetry IIA & $\theta_{23} \rightarrow-\theta_{23}, \theta_{12} \rightarrow-\theta_{12}$ & \\
\hline Symmetry IIB & $\theta_{23} \rightarrow-\theta_{23}, \delta \rightarrow \delta+\pi$ & \\
\hline Symmetry IIIA & $\theta_{13} \rightarrow-\theta_{13}, \theta_{12} \rightarrow-\theta_{12}$ & \\
\hline Symmetry IIIB & $\theta_{13} \rightarrow-\theta_{13}, \delta \rightarrow \delta+\pi$ & \\
\hline Symmetry IVA & $\theta_{23} \rightarrow-\theta_{23}, \theta_{13} \rightarrow-\theta_{13}$ & \\
\hline Symmetry IVB & $\begin{array}{c}\theta_{23} \rightarrow-\theta_{23}, \theta_{13} \rightarrow-\theta_{13} \\
\theta_{12} \rightarrow-\theta_{12}, \delta \rightarrow \delta+\pi\end{array}$ & \\
\hline
\end{tabular}

symmetry, is also given in Ref. [12]. An alternative view of these symmetries as due to rephasing invariance of the $S$ matrix is presented in Ref. [13]. In this paper, we will shed more light on the nature of these relabeling or reparametrization symmetries by bringing the new symmetries in DMP into this context. Through a renewed discussion on symmetry from the Hamiltonian point of view, we will be able to sharpen up our previous statement on the Hamiltonian symmetry.

We start our discussion from what we call the "symmetry finder" (SF) equation in vacuum [14]. It is nothing but the expression of the flavor basis state (i.e., wave function) $\nu$ in terms of the mass eigenstate $\breve{\nu}$ in the following two different ways:

$$
\nu=U\left(\theta_{23}, \theta_{13}, \theta_{12}, \delta\right) \check{\nu}=U\left(\theta_{23}^{\prime}, \theta_{13}^{\prime}, \theta_{12}^{\prime}, \delta^{\prime}\right) \check{\nu}^{\prime},
$$

where the quantities with "prime" imply the transformed ones and $\breve{\nu}^{\prime}$ may involve eigenstate exchanges and/or rephasing of the wave functions. Since the SF equation represents the same flavor state by the two different sets of the physical parameters, it implies a symmetry. See Sec. III for more details.

We utilize the SF equation in its generalized form in matter to uncover new symmetries associated with the mass-eigenstate exchange $1 \leftrightarrow 2$. We first treat the exact solution of neutrino oscillation under a uniform matter density, the Zaglauer-Schwarzer system [15] in Sec. IV. Then, as the highlight of the SF symmetry discussion, it will be applied to the DMP framework [11] in Secs. V and VI. The DMP perturbation theory gives an approximate but very accurate description of neutrino oscillation in matter with uniform matter density [16], and at the same time displays a clear physical picture that covers the entire kinematical region of the terrestrial neutrino experiments [13]. In fact, we will uncover the eight symmetries whose seven are new, with interesting varying features of the mixed fundamental and dynamical symmetries. See the summary Table I in Sec. V H. Because of the frequent usage of the term such as "the DMP system" or "the DMP framework," we sometimes abbreviate them as just "DMP" hereafter.

A few words on the type of symmetry we are going to discuss in this paper. It does not involve the transformations that connect our world to the other one, such as $\Delta m_{31}^{2} \rightarrow-\Delta m_{31}^{2}$, or $\theta_{12} \leq \frac{\pi}{4}$ to $\theta_{12} \geq \frac{\pi}{4}{ }^{2}$. By restricting the symmetry transformation into the ones that keep the system to remain in our world, it inevitably takes the form of reparametrization or relabeling symmetry. Implications of the newly observed reparametrization symmetry are discussed in Secs. VI and VII.

\section{THE THREE NEUTRINO EVOLUTION IN MATTER IN THE $\nu$ SM}

In this paper, we restrict ourselves into discussions within the neutrino-mass-embedded Standard Model, $\nu \mathrm{SM}$ for short. We discuss symmetry that exists in the oscillation probability in the standard three-flavor neutrino system defined by the Hamiltonian in the flavor basis

$$
H=\frac{1}{2 E}\left\{U\left[\begin{array}{ccc}
m_{1}^{2} & 0 & 0 \\
0 & m_{2}^{2} & 0 \\
0 & 0 & m_{3}^{2}
\end{array}\right] U^{\dagger}+\left[\begin{array}{ccc}
a(x) & 0 & 0 \\
0 & 0 & 0 \\
0 & 0 & 0
\end{array}\right]\right\},
$$

\footnotetext{
${ }^{2}$ In contrast, it is argued in Ref. [17] that the sign- $\Delta m^{2}$ and the $\theta_{23}$ octant degeneracies can be understood as a consequence of the symmetries, though approximate ones, which connect the systems with differing signs of $\Delta m_{31}^{2}$ [8] and the worlds with $\theta_{23} \leq \frac{\pi}{4}$ and $\theta_{23} \geq \frac{\pi}{4}[18]$.
} 
where $E$ is the neutrino energy and $m_{i}(i=1,2,3)$ the neutrino masses of $i$ th mass eigenstate. In Eq. (2.1), $U \equiv$ $U_{\text {MNS }}$ denotes the standard $3 \times 3$ lepton flavor mixing matrix [19] which relates the flavor neutrino states to the vacuum mass eigenstates as $\nu_{\alpha}=U_{\alpha i} \nu_{i}$, where $\alpha$ runs over $e, \mu, \tau$, and the mass eigenstate index $i$ runs over 1,2, and 3 . The functions $a(x)$ in (2.1) denote the Wolfenstein matter potential [20] due to charged current (CC) reactions

$a(x)=2 \sqrt{2} G_{F} N_{e} E \approx 1.52 \times 10^{-4}\left(\frac{Y_{e} \rho(x)}{\mathrm{g} \mathrm{cm}^{-3}}\right)\left(\frac{E}{\mathrm{GeV}}\right) \mathrm{eV}^{2}$.

Here, $G_{F}$ is the Fermi constant, and $N_{e}$ is the electron number density in matter. $\rho(x)$ and $Y_{e}$ denote, respectively, the matter density and number of electrons per nucleon in matter.

In Secs. IV and V, our treatment assumes the uniform matter density approximation. Whereas in Sec. VI where we discuss the Hamiltonian view of the symmetries, we get rid of this restriction but keep variation of matter density sufficiently mild such that the adiabatic approximation applies.

\section{A. The SOL convention of the flavor mixing matrix}

We use, throughout this paper, the solar (SOL) convention $[14,21]$ for the lepton mixing matrix

$$
\begin{aligned}
U_{\mathrm{SOL}}= & {\left[\begin{array}{ccc}
1 & 0 & 0 \\
0 & c_{23} & s_{23} \\
0 & -s_{23} & c_{23}
\end{array}\right]\left[\begin{array}{ccc}
c_{13} & 0 & s_{13} \\
0 & 1 & 0 \\
-s_{13} & 0 & c_{13}
\end{array}\right] } \\
& \times\left[\begin{array}{ccc}
c_{12} & s_{12} e^{i \delta} & 0 \\
-s_{12} e^{-i \delta} & c_{12} & 0 \\
0 & 0 & 1
\end{array}\right] \\
\equiv & U_{23}\left(\theta_{23}\right) U_{13}\left(\theta_{13}\right) U_{12}\left(\theta_{12}, \delta\right),
\end{aligned}
$$

in which $e^{ \pm i \delta}$ is attached to the sine of the "solar angle" $\theta_{12}$, with $\delta$ being the lepton $C P$ phase. We note that $s_{i j} \equiv \sin \theta_{i j}$, etc., are the common abbreviated notations. We will see in Sec. III that the SOL convention is the most convenient one to discuss the symmetries that involve the 1-2 mass eigenstate exchange and the $C P$ phase $\delta$. With the SOL convention, we obtain exactly the same oscillation probability as the one calculated by using the well known Particle Data Group (PDG) [22] convention, as to be seen immediately below. Thus, there is no reason for being afraid of using the SOL convention, despite its unfamiliar status. $^{3}$

The mixing matrix in the SOL and the PDG conventions are related by [21]

\footnotetext{
${ }^{3}$ Yet, the SOL convention of the mixing matrix has been used in an analysis of the parameter correlations in the theory with unitarity violation [23].
}

$U_{\mathrm{SOL}}=\left[\begin{array}{ccc}1 & 0 & 0 \\ 0 & e^{-i \delta} & 0 \\ 0 & 0 & e^{-i \delta}\end{array}\right] U_{\mathrm{PDG}}\left[\begin{array}{ccc}1 & 0 & 0 \\ 0 & e^{i \delta} & 0 \\ 0 & 0 & e^{i \delta}\end{array}\right]$

Then, their flavor-basis Hamiltonian and the neutrino states in both conventions are related to each other by

$$
\begin{aligned}
H_{\mathrm{SOL}} & =\left[\begin{array}{ccc}
1 & 0 & 0 \\
0 & e^{-i \delta} & 0 \\
0 & 0 & e^{-i \delta}
\end{array}\right] H_{\mathrm{PDG}}\left[\begin{array}{ccc}
1 & 0 & 0 \\
0 & e^{i \delta} & 0 \\
0 & 0 & e^{i \delta}
\end{array}\right], \\
\nu_{\mathrm{SOL}} & =\left[\begin{array}{ccc}
1 & 0 & 0 \\
0 & e^{-i \delta} & 0 \\
0 & 0 & e^{-i \delta}
\end{array}\right] \nu_{\mathrm{PDG}},
\end{aligned}
$$

where $H_{\mathrm{SOL}}$ and $\nu_{\mathrm{SOL}}\left(H_{\mathrm{PDG}}\right.$ and $\left.\nu_{\mathrm{PDG}}\right)$ denote the Hamiltonian written with the use of the $U$ matrix of the SOL (PDG) convention and the neutrino flavor state under the convention. The $S$ matrix obeys the same relation as that of the Hamiltonian in Eq. (2.5). It means that both the Hamiltonian and the $S$ matrix are identical up to the phase redefinition of neutrino wave functions. Therefore, the oscillation probability calculated with $H_{\text {SOL }}$ is identical with the one obtained with the usual PDG convention $U$ matrix, as stated above.

In Sec. V we will face another convention change, the atmospheric (ATM) to the SOL conventions of the $U$ matrix, where $e^{ \pm i \delta}$ is attached to $s_{23}$ in the ATM convention. Therefore, we give here the corresponding transformation rule between the ATM and SOL conventions [21]:

$$
\begin{aligned}
H_{\mathrm{SOL}} & =\left[\begin{array}{ccc}
1 & 0 & 0 \\
0 & e^{-i \delta} & 0 \\
0 & 0 & 1
\end{array}\right] H_{\mathrm{ATM}}\left[\begin{array}{ccc}
1 & 0 & 0 \\
0 & e^{i \delta} & 0 \\
0 & 0 & 1
\end{array}\right], \\
\nu_{\mathrm{SOL}} & =\left[\begin{array}{ccc}
1 & 0 & 0 \\
0 & e^{-i \delta} & 0 \\
0 & 0 & 1
\end{array}\right] \nu_{\mathrm{ATM}} .
\end{aligned}
$$

The ATM convention of the $U$ matrix is used in Refs. $[11,13,21,24]$, and the references cited therein.

\section{SYMMETRY FINDER FOR NEUTRINO OSCILLATION IN VACUUM}

\section{A. Symmetry finder equation}

In vacuum the flavor eigenstate $\nu$ is related to the mass eigenstate $\hat{\nu}$ as

$$
\nu=U_{23}\left(\theta_{23}\right) U_{13}\left(\theta_{13}\right) U_{12}\left(\theta_{12}, \delta\right) \hat{\nu} .
$$

Using the SOL convention $U$ matrix in Eq. (2.3), one can easily prove the relation [14] 


$$
\begin{aligned}
U_{12}\left(\theta_{12}, \delta\right)\left[\begin{array}{l}
\nu_{1} \\
\nu_{2} \\
\nu_{3}
\end{array}\right] & =U_{12}\left(\theta_{12}+\frac{\pi}{2}, \delta\right)\left[\begin{array}{c}
-e^{i \delta} \nu_{2} \\
e^{-i \delta} \nu_{1} \\
\nu_{3}
\end{array}\right] \\
& =U_{12}\left(\frac{\pi}{2}-\theta_{12}, \delta \pm \pi\right)\left[\begin{array}{c}
e^{i \delta} \nu_{2} \\
-e^{-i \delta} \nu_{1} \\
\nu_{3}
\end{array}\right] .
\end{aligned}
$$

We show below that this equation is a powerful tool for uncovering symmetry in neutrino oscillation in a vacuum. For this reason, we denote Eq. (3.2) and its extension in matter as the SF equation.

It is easy to observe that the relation (3.2) implies symmetry [14]. The first equality means that the use of $\theta_{12}^{\prime}=\theta_{12}+\frac{\pi}{2}$ and the exchanged (and rephased) mass eigenstates $1 \leftrightarrow 2$ produces the same oscillation probability. Since rephasing does not affect the observables, the first equality in Eq. (3.2) implies the $1 \leftrightarrow 2$ exchange symmetry under the transformation

Symmetry IA-vacuum: $m_{1}^{2} \leftrightarrow m_{2}^{2}, \quad c_{12} \rightarrow-s_{12}, \quad s_{12} \rightarrow c_{12}$,

where the existence of an alternative choice, $c_{12} \rightarrow s_{12}$ and $s_{12} \rightarrow-c_{12}\left(\theta_{12} \rightarrow \theta_{12}-\frac{\pi}{2}\right)$, is understood. Similarly, the second equality in (3.2) implies the symmetry of the probability under the transformation

Symmetry IB-vacuum: $m_{1}^{2} \leftrightarrow m_{2}^{2}, \quad c_{12} \leftrightarrow s_{12}, \quad \delta \rightarrow \delta \pm \pi$.

Throughout this paper we will observe pairing of the symmetries, the types "A" and "B," where A does not contain $\delta$, while $\mathrm{B}$ does. The question of how the pair should be chosen in the presence of many symmetries will be answered in the treatment of the DMP perturbation theory in Secs. V and VI, in which we will observe eight symmetries.

There are many ways to confirm that the transformations of symmetries IA and IB above leave the oscillation probability invariant. One can do it by using the explicit expressions of the probability in vacuum. One can also show the invariance of the $S$ matrix elements [14] or the invariance of the vacuum part of the Hamiltonian (2.1). ${ }^{4}$ See the comment at the end of Sec. VIC. The last path, finding a symmetry by the SF equation followed by confirmation of its validity by examining the

\footnotetext{
${ }^{4}$ In fact, the symmetry IA-vacuum leaves the whole Hamiltonian $H$ in Eq. (2.1) invariant, as the matter potential term does not transform. Using this feature the author of Ref. [25] discussed how one can organize the perturbative expansion in such a way that the symmetry is respected in each order.
}

Hamiltonian, anticipates the route we will take in our treatment of the symmetries in DMP.

\section{B. Clarification and interpretation of symmetries IA and IB in vacuum}

Now a clarifying remark must be made: The notation $\theta_{12}^{\prime}=\theta_{12}+\frac{\pi}{2}$, which is used for simplicity of expression of Eq. (3.2), may be confusing because it requires one to expand the region of definition of $\theta_{12}$ outside of what is usually taken, $0 \leq \theta_{12} \leq \frac{\pi}{2}$ [22]. While it is possible, we do not choose this option in this paper. What we have meant for symmetry IA is, therefore, an invariance under the transformation in which $c_{12}$ is replaced by $-s_{12}$, and $s_{12}$ is replaced by $c_{12}$ simultaneously with the $1 \leftrightarrow 2$ state exchange $m_{1}^{2} \leftrightarrow m_{2}^{2}$ under the condition that both the initial and the transformed $\theta_{12}$ remain in the region $0 \leq \theta_{12} \leq \frac{\pi}{2}$. We apply this principle to all the mass eigenstate exchange symmetries $1 \leftrightarrow 2$ in this paper. As a matter of fact, we restrict ourselves to the region of $\theta_{12}$ as $0 \leq \theta_{12} \leq \frac{\pi}{4}$, the favored region [26] first observed experimentally by Davis et al. [27]. Under this prescription, the symmetry IB in Eq. (3.4) has nothing to do with the socalled "dark-side" discussion $[28,29]$.

Then the question would be: What is the interpretation of symmetries IA and IB in a vacuum? The answer is that the SF equation (3.2) offers three different descriptions of the unique (i.e., identical) world. If one computes the flavor basis neutrino wave functions by using Eq. (3.1) with the three different ways of the 1-2 space rotation, one obtains the identical expressions of $\nu_{e}, \nu_{\mu}$, and $\nu_{\tau}$ in terms of the three mass eigenstate components $\nu_{1}, \nu_{2}$, and $\nu_{3}$. Therefore, symmetries IA and IB are both the relabeling or reparametrization symmetries $1 \leftrightarrow 2$ associated with the transformations of $\theta_{12}$ and possibly $\delta$. It is intriguing to see whether the similar pairing structure of types A and B prevails in matter.

\section{SYMMETRY FINDER FOR NEUTRINO OSCILLATION IN MATTER}

In this and the following two sections, we extend the symmetry finding framework in neutrino oscillation, symmetry finder, into the matter environments. In neutrino oscillation in matter, we often encounter the situation that all or some of the mixing angles and the $C P$ phase $\delta$ becomes matter dressed. This is the case in the systems we will treat, all become matter dressed in the ZaglauerSchwarzer (ZS) system, and only $\theta_{12}$ and $\theta_{13}$ are altered to matter-affected ones in DMP. For notational simplicity and unity we denote the changes in notations for the mixing parameters from vacuum to matter-dressed ones as

$\theta_{12} \rightarrow \psi, \quad \theta_{13} \rightarrow \phi, \quad \theta_{23} \rightarrow \eta, \quad \delta \rightarrow \tilde{\delta}$, 
throughout Secs. IV-VI, except that $\theta_{23}$ and $\delta$ are kept matter undressed in the latter two sections. Hereafter, we use the abbreviated notations $c_{\psi} \equiv \cos \psi, s_{\phi} \equiv \sin \phi$, etc.

\section{A. The Zaglauer-Schwarzer system}

Now we apply the SF method to the ZS system in this section as a simple extension of the SF equation in vacuum. The authors of Ref. [15] exactly solved the three-flavor neutrino evolution in matter with uniform density. The flavor neutrino eigenstate is related to the matter propagation eigenstate $\hat{v}$ in the SOL convention as

$$
\nu=U_{23}(\eta) U_{13}(\phi) U_{12}(\psi, \tilde{\delta}) \hat{\nu} .
$$

Then, the expressions of the oscillation probability in various channels in matter can be obtained by doing the replacement given in Eq. (4.1) in the formulas in vacuum. The expressions of the matter-dressed mixing angles and the $C P$ phase are given in Ref. [15]. Though they are given in the PDG convention, one can easily translate them into the ones in the SOL convention by using Eq. (2.4).

The exact expressions of the three eigenvalues of $2 E H$ are obtained in Ref. [30], which are denoted here as $\lambda_{i}$ $(i=1,2,3)$. Our notation is such that, in the normal mass ordering, $\lambda_{3}>\lambda_{2}>\lambda_{1}$ in the region of a large positive matter potential defined in Eq. (2.2). This prescription for defining $\lambda_{i}$ will be used also in the discussion of the DMP system in Sec. V. Thanks to the complete parallelism between neutrino oscillations in vacuum and in matter we can immediately conclude, by using the SF equation in matter with replacement (4.1) in Eq. (3.2), that the matter versions of symmetry IA and symmetry IB in the ZS system exist:

Symmetry IA-ZS : $\quad \lambda_{1} \leftrightarrow \lambda_{2}, \quad c_{\psi} \rightarrow-s_{\psi}, \quad s_{\psi} \rightarrow c_{\psi}$, Symmetry IB-ZS : $\quad \lambda_{1} \leftrightarrow \lambda_{2}, \quad c_{\psi} \leftrightarrow s_{\psi}, \quad \tilde{\delta} \rightarrow \tilde{\delta} \pm \pi$.

Notice that these are the symmetries whose transformations consist only of the matter-dressed variables. None of the fundamental parameters, the ones in the original Hamiltonian in Eq. (2.1), transform.

\section{B. Fundamental vs dynamical symmetries}

A clarifying remark is in order on our terminology: In this paper, we often use the terms, "fundamental variables" and "dynamical variables." The fundamental variables are meant to be the mixing parameters in the flavor basis Hamiltonian in Eq. (2.1). In contrast, dynamical variables are characterized as the variables by which the Hamiltonian is diagonalized exactly (ZS case) or approximately (DMP case) [13]. In the systems discussed in this paper, therefore, the dynamical variables are the matter-dressed variables.
A dynamical symmetry is the symmetry without its trace in the Hamiltonian of the system, but the one which indeed arises after the system is solved. Hence, it often happens that the dynamical symmetry has transformations written by the variables that are used to diagonalize the Hamiltonian. On the contrary, if the symmetry is described solely by the fundamental variables, it is called the fundamental symmetry. We will see later that most of the symmetries in DMP have the mixed fundamental-dynamical character.

\section{Generic $\boldsymbol{i} \leftrightarrow \boldsymbol{j}$ state exchange symmetry?}

A natural question would be: Why should we restrict ourselves to the $1 \leftrightarrow 2$ exchange symmetry? How about a $1 \leftrightarrow 3$ exchange symmetry, for example? It is a very good point, but we do not know a complete answer about the feasibility of such an extension.

Suppose that we take a different parametrization of the $U$ matrix such as $U=U_{23}\left(\theta_{23}^{\prime}\right) U_{12}\left(\theta_{12}^{\prime}\right) U_{13}\left(\theta_{13}^{\prime}, \delta^{\prime}\right)$ by exchanging the order of $U_{13}$ and $U_{12}$ rotations (see, e.g., Ref. [31]). Then, the similar treatment can go through in vacuum and in matter, which would lead to an analogous discussion of the $1 \leftrightarrow 3$ exchange symmetry. But, $\theta_{i j}^{\prime}(i$, $j=1,2,3)$ and $\delta^{\prime}$ in this new parametrization is completely different from those in our conventional parametrization. Rewriting the newly obtained $1 \leftrightarrow 3$ exchange symmetry transformations written with $\theta_{i j}^{\prime}(i, j=1,2,3)$ and $\delta^{\prime}$ by the three angles and $C P$ phase in our SOL or PDG convention $U$ matrix would be a formidable task.

Therefore, in principle, one could discuss the $1 \leftrightarrow 3$ or $2 \leftrightarrow 3$ exchange symmetries in the same way as we do, but no useful output is expected if we write the symmetry transformations by the angles and the $C P$ phase in the $U$ matrix of our usual conventions. For this reason we restrict our discussions to the $1 \leftrightarrow 2$ exchange symmetry in this paper.

\section{SYMMETRY FINDER IN THE DMP SYSTEM}

We now discuss the SF equation and its application to the $1 \leftrightarrow 2$ eigenstate exchange symmetry in the Denton et al. framework [11]. Our treatment in this section is valid to first order in the DMP perturbation theory. Later in Sec. VI, we discuss the Hamiltonian proof of the symmetry, which, in fact, guarantees its validity to all orders.

After brief recollections of the DMP perturbation theory in Secs. VA and V B, we introduce the SF equation in DMP in Sec. V C by taking into account the possible rephasing of both the neutrino flavor and the energy eigenstates. By using the SF equation we will find the eight symmetries in DMP, which we call symmetry I-, II-, III-, and IV-DMP doubled with the pairing of "A" (no $\delta$ ) and "B" (with $\delta$ ) types. All eight symmetries are tabulated in the summary Table I in section V H. All of them are the reparametrization symmetries with the $1 \leftrightarrow 2$ state exchange. Invariance of the oscillation probability under the transformations listed in Table I is verified explicitly by using the probability 
formulas given in Refs. [11,13], which is, however, left for the readers as a simple exercise. ${ }^{5}$

\section{A. DMP at the zeroth order}

At the leading (zeroth) order in the DMP perturbation theory, the flavor neutrino eigenstate is related to the propagation eigenstate in matter as [11]

$$
\nu=U_{23}\left(\theta_{23}\right) U_{13}(\phi) U_{12}(\psi, \delta) \hat{\nu},
$$

which is identical with the vacuum form (3.1) apart from replacements $\theta_{12} \rightarrow \psi$ and $\theta_{13} \rightarrow \phi$. Or, starting from Eq. (4.2) in the ZS system, $\eta$ and $\tilde{\delta}$ take the vacuum values $\theta_{23}$ and $\delta$. It must be a good approximation, given that the matter effect modification is modest for them [15,32]. Then, at the leading order, the DMP system possesses the symmetries akin to symmetries IA-ZS and IB-ZS in Eq. (4.3), but with $\eta$ and $\tilde{\delta}$ replaced by $\theta_{23}$ and $\delta$, respectively.

\section{B. DMP expansion to first order}

However, when the first order correction is added, the structure of the SF equation changes. It can be obtained most easily by the so-called $V$ matrix method [33] as done in Ref. [11], which takes the form in the SOL convention as ${ }^{6}$

$$
\begin{aligned}
{\left[\begin{array}{c}
\nu_{e} \\
\nu_{\mu} \\
\nu_{\tau}
\end{array}\right]=} & U_{23}\left(\theta_{23}\right) U_{13}(\phi) U_{12}(\psi, \delta) \\
& \times\left\{1+\epsilon c_{12} s_{12} \sin \left(\phi-\theta_{13}\right) \mathcal{W}\left(\psi, \delta ; \lambda_{1}, \lambda_{2}\right)\right\}\left[\begin{array}{l}
\nu_{1} \\
\nu_{2} \\
\nu_{3}
\end{array}\right],
\end{aligned}
$$

where $\mathcal{W}\left(\psi, \delta ; \lambda_{1}, \lambda_{2}\right)$ is defined by

$$
\mathcal{W}\left(\psi, \delta ; \lambda_{1}, \lambda_{2}\right) \equiv\left[\begin{array}{ccc}
0 & 0 & -s_{\psi} \frac{\Delta m_{\text {ren }}^{2}}{\lambda_{3}-\lambda_{1}} \\
0 & 0 & c_{\psi} e^{-i \delta \frac{\Delta m_{\text {ren }}^{2}}{\lambda_{3}-\lambda_{2}}} \\
s_{\psi} \frac{\Delta m_{\text {ren }}^{2}}{\lambda_{3}-\lambda_{1}} & -c_{\psi} e^{i \delta \frac{\Delta m_{\text {ren }}^{2}}{\lambda_{3}-\lambda_{2}}} & 0
\end{array}\right] .
$$

In Eqs. (5.2) and (5.3), the notations $\phi$ and $\psi$ are defined in Eq. (4.1), and their expressions are given in Ref. [11]. $\epsilon$ is the

\footnotetext{
${ }^{5}$ We recommend arXiv version 3 of Ref. [13] for more explicitly written formulas for verification.

${ }^{6}$ In the original reference [11], the expression of this SF equation (5.2) is given in the ATM convention defined in Sec. II A. The expression in Eq. (5.2) with (5.3) in the SOL convention can be obtained by the transformation in Eq. (2.6) in Sec. II A.
}

unique expansion parameter in the DMP perturbation theory and is defined as

$$
\epsilon \equiv \frac{\Delta m_{21}^{2}}{\Delta m_{\mathrm{ren}}^{2}}, \quad \Delta m_{\mathrm{ren}}^{2} \equiv \Delta m_{31}^{2}-s_{12}^{2} \Delta m_{21}^{2},
$$

where $\Delta m_{\mathrm{ren}}^{2}$ is the "renormalized" atmospheric $\Delta m^{2}$ introduced in Ref. [24]. With this added structure that comes from first-order corrections, we need a reformulation of our symmetry hunting scheme using the SF equation.

\section{Symmetry finder equation in DMP}

In looking for the solution to the SF equation (5.2) we introduce the ansatz

$$
\begin{aligned}
F\left[\begin{array}{c}
\nu_{e} \\
\nu_{\mu} \\
\nu_{\tau}
\end{array}\right]= & F U_{23}\left(\theta_{23}\right) U_{13}(\phi) U_{12}(\psi, \delta) G^{\dagger} G \\
& \times\left\{1+\epsilon c_{12} s_{12} \sin \left(\phi-\theta_{13}\right) \mathcal{W}\left(\psi, \delta ; \lambda_{1}, \lambda_{2}\right)\right\} \\
& \times G^{\dagger} G\left[\begin{array}{l}
\nu_{1} \\
\nu_{2} \\
\nu_{3}
\end{array}\right],
\end{aligned}
$$

for an alternative expression of the state, analogously as in Eq. (3.2). In Eq. (5.5) we have introduced the flavor-state rephasing matrix $F$, which is defined by

$$
F \equiv\left[\begin{array}{ccc}
e^{i \tau} & 0 & 0 \\
0 & e^{i \sigma} & 0 \\
0 & 0 & 1
\end{array}\right],
$$

and the generalized $1 \leftrightarrow 2$ state exchange matrix $G$

$$
\begin{aligned}
G & \equiv\left[\begin{array}{ccc}
0 & -e^{i(\delta+\alpha)} & 0 \\
e^{-i(\delta+\beta)} & 0 & 0 \\
0 & 0 & 1
\end{array}\right], \\
G^{\dagger} & \equiv\left[\begin{array}{ccc}
0 & e^{i(\delta+\beta)} & 0 \\
-e^{-i(\delta+\alpha)} & 0 & 0 \\
0 & 0 & 1
\end{array}\right],
\end{aligned}
$$

where $\tau, \sigma, \alpha$, and $\beta$ denote the arbitrary phases. Notice that the rephasing matrices, both $F$ and $G$ in Eqs. (5.6) and (5.7), take the nonvanishing, nontrivial (not unity) elements in the 1-2 subsector. It is because we restrict ourselves into the $1 \leftrightarrow 2$ state exchange symmetry.

Now, we call the readers' attention to the following: since we have introduced the flavor-state rephasing matrix $F, \theta_{23}$ and $\phi$, in addition to $\delta$, in principle, must transform, as indicated in the explicit form of the SF equation (5.5), 


$$
\begin{aligned}
& {\left[\begin{array}{ccc}
e^{i \tau} & 0 & 0 \\
0 & e^{i \sigma} & 0 \\
0 & 0 & 1
\end{array}\right]\left[\begin{array}{l}
\nu_{e} \\
\nu_{\mu} \\
\nu_{\tau}
\end{array}\right]=\left[\begin{array}{ccc}
1 & 0 & 0 \\
0 & c_{23} & s_{23} e^{i \sigma} \\
0 & -s_{23} e^{-i \sigma} & c_{23}
\end{array}\right]\left[\begin{array}{ccc}
c_{\phi} & 0 & s_{\phi} e^{i \tau} \\
0 & 1 & 0 \\
-s_{\phi} e^{-i \tau} & 0 & c_{\phi}
\end{array}\right]\left[\begin{array}{ccc}
c_{\psi} & s_{\psi} e^{i(\delta+\tau-\sigma)} & 0 \\
-s_{\psi} e^{-i(\delta+\tau-\sigma)} & c_{\psi} & 0 \\
0 & 0 & 1
\end{array}\right]\left[\begin{array}{ccc}
e^{i \tau} & 0 & 0 \\
0 & e^{i \sigma} & 0 \\
0 & 0 & 1
\end{array}\right] G^{\dagger}} \\
& \times G\left\{1+\epsilon c_{12} s_{12} \sin \left(\phi-\theta_{13}\right) \mathcal{W}\left(\psi, \delta ; \lambda_{1}, \lambda_{2}\right)\right\} G^{\dagger} G\left[\begin{array}{l}
\nu_{1} \\
\nu_{2} \\
\nu_{3}
\end{array}\right] \text {. }
\end{aligned}
$$

Notice that rephasing not only of the flavor states $\nu_{\alpha}$ $(\alpha=e, \mu, \tau)$ but also the matter eigenstates $\nu_{j}(j=1,2,3)$ does not affect the oscillation probability.

For $s_{23}^{\prime}$ and $s_{\phi}^{\prime}$, we restrict ourselves to the simple solutions $s_{23} e^{i \sigma}=s_{23}^{\prime}$ and $s_{\phi} e^{i \tau}=s_{\phi}^{\prime}$. Apparently, there is no other way as far as we remain in the present formulation of the SF equation (5.8). Once we place this restriction, we have to limit the possible solutions of $\tau$ and $\sigma$ to integer multiples of $\pi$, since otherwise we have to make the mixing angles complex. We will see in the next Sec. V D that it has a tremendous consequence to restrict the solution space of the SF equation.
Under the ansatz $s_{23} e^{i \sigma}=s_{23}^{\prime}$ and $s_{\phi} e^{i \tau}=s_{\phi}^{\prime}$, the SF equation (5.8) can be decomposed into the following first and second conditions: The first condition reads

$$
\begin{aligned}
& {\left[\begin{array}{ccc}
c_{\psi} & s_{\psi} e^{i(\delta+\tau-\sigma)} & 0 \\
-s_{\psi} e^{-i(\delta+\tau-\sigma)} & c_{\psi} & 0 \\
0 & 0 & 1
\end{array}\right]\left[\begin{array}{ccc}
e^{i \tau} & 0 & 0 \\
0 & e^{i \sigma} & 0 \\
0 & 0 & 1
\end{array}\right] G^{\dagger}} \\
& =U_{12}\left(\psi^{\prime}, \delta+\xi\right),
\end{aligned}
$$

while the second condition takes the form

$$
\begin{gathered}
\epsilon c_{12} s_{12} \sin \left(\phi-\theta_{13}\right)\left[\begin{array}{ccc}
0 & 0 & -c_{\psi} e^{i \alpha} \frac{\Delta m_{\mathrm{ren}}^{2}}{\lambda_{3}-\lambda_{2}} \\
0 & 0 & -s_{\psi} e^{-i(\delta+\beta)} \frac{\Delta m_{\mathrm{ren}}^{2}}{\lambda_{3}-\lambda_{1}} \\
c_{\psi} e^{-i \alpha} \frac{\Delta m_{\mathrm{ren}}^{2}}{\lambda_{3}-\lambda_{2}} & s_{\psi} e^{i(\delta+\beta) \frac{\Delta m_{\mathrm{ren}}^{2}}{\lambda_{3}-\lambda_{1}}} & 0
\end{array}\right] \\
=\epsilon c_{12}^{\prime} s_{12}^{\prime} \sin \left(\phi^{\prime}-\theta_{13}^{\prime}\right)\left[\begin{array}{ccc}
0 & 0 & -s_{\psi}^{\prime} \frac{\Delta m_{\mathrm{ren}}^{2}}{\lambda_{3}-\lambda_{2}} \\
0 & 0 & c_{\psi}^{\prime} e^{-i(\delta+\xi)} \frac{\Delta m_{\mathrm{ren}}^{2}}{\lambda_{3}-\lambda_{1}} \\
s_{\psi}^{\prime} \frac{\Delta m_{\mathrm{ren}}^{2}}{\lambda_{3}-\lambda_{2}} & -c_{\psi}^{\prime} e^{i(\delta+\xi)} \frac{\Delta m_{\mathrm{ren}}^{2}}{\lambda_{3}-\lambda_{1}} & 0
\end{array}\right] .
\end{gathered}
$$

\section{How to solve the DMP SF equation?}

We analyze the first condition (5.9). It is not difficult to show that it entails the conditions

$$
\begin{aligned}
& c_{\psi^{\prime}}=-s_{\psi} e^{-i(\alpha-\tau)}=-s_{\psi} e^{i(\beta+\sigma)}, \\
& s_{\psi^{\prime}}=c_{\psi} e^{i(\beta+\tau-\xi)}=c_{\psi} e^{-i(\alpha-\sigma-\xi)},
\end{aligned}
$$

and the consistency conditions for the phases

$\alpha+\beta-\tau+\sigma=0 \quad(\bmod 2 \pi), \quad \tau-\sigma-\xi=0, \pm \pi$,

where all the solutions of the phases are modulo $2 \pi$. In the second equation in Eq. (5.12), a classification naturally appeared:
Class I: $\tau-\sigma=\xi$, Class II: $\tau-\sigma=\xi \pm \pi$.

Since we have restricted $\tau$ and $\sigma$ to integer multiples of $\pi$, Eq. (5.11) dictates that $\alpha, \beta$, and $\xi$ must also be integer multiples of $\pi$. That is, all the $\xi, \tau, \sigma, \alpha$, and $\beta$ must be integer multiples of $\pi$. Then, the procedure for obtaining solutions to the SF equation is as follows:

(i) To choose an ansatz for $\xi$. In this paper we try only the limited choices, $\xi=0, \pi$.

(ii) To choose class I or class II. Then, find all possible solutions for $\tau, \sigma, \alpha$, and $\beta$.

(iii) Verify the solution against the second condition (5.10).

It is interesting to observe that the A-type and B-type pairings naturally arise from the choices $\xi=0$ or $\pi$, which ultimately came from the first condition in the SF equation (5.8). This and the other consequences will be 
explained in Sec. VH. Though we do not know if the solutions are complete, we did not make any serious effort to go outside of the current framework. ${ }^{7}$

\section{E. Symmetries IA and IB in DMP}

Let us analyze the SF equation in Eqs. (5.9) and (5.10). We first recover the $\lambda_{1} \leftrightarrow \lambda_{2}$ exchange symmetry, the one found in the original DMP work [11], which is denoted as "symmetry IA-DMP" for the classification purpose:

Symmetry IA-DMP : $\lambda_{1} \leftrightarrow \lambda_{2}, \quad c_{\psi} \rightarrow \mp s_{\psi}, \quad s_{\psi} \rightarrow \pm c_{\psi}$.

Since no transformation of the fundamental variables is involved, we can set $\tau=\sigma=0$. Following the prescription in Sec. V D we try the two cases, $\xi=0$ and $\xi=\pi$. Notice that $\xi$ is the parameter that causes a possible shift of $\delta$ in the transformed system, as in Eq. (5.9).

\section{Case of $\xi=0$ : Class I}

It is easy to observe, with Eqs. (5.11) and (5.12), that there are two solutions of $\alpha$ and $\beta, \alpha=\beta=0$ for the upper sign and $\alpha=\pi, \beta=-\pi$ for the lower sign in Eq. (5.14), respectively. The consistency with the second condition (5.10) can easily be checked. Therefore, it is shown that symmetry IA-DMP in Eq. (5.14) is the solution to the SF equation in DMP.

\section{Case of $\xi=\pi$ : Class II}

Let us explore the case of $\xi=\pi$ to look for a new solution. If we try the cases of $\alpha=\pi, \beta=-\pi$, and $\alpha=\beta=0$, we obtain $c_{\psi^{\prime}}=s_{\psi}, s_{\psi^{\prime}}=c_{\psi} \quad\left(c_{\psi^{\prime}}=-s_{\psi}\right.$, $s_{\psi^{\prime}}=-c_{\psi}$ ) for the former (latter) case. Then, the second condition tells us that the sign flip of $s_{12}$ is required. Thus, we have found a new symmetry "symmetry IB-DMP" as a solution to the SF equation:

Symmetry IB-DMP : $\theta_{12} \rightarrow-\theta_{12}, \quad \delta \rightarrow \delta+\pi$,

$$
\lambda_{1} \leftrightarrow \lambda_{2}, \quad c_{\psi} \rightarrow \pm s_{\psi}, \quad s_{\psi} \rightarrow \pm c_{\psi} .
$$

Thus, we have found that the pairing structure of the $1 \leftrightarrow 2$ exchange symmetry with $\mathrm{A}$ ( $\delta$ free) and B (with $\delta$ ) types prevails in DMP.

A question might be: After we find more symmetries, which will be the case, why is the pairing of symmetries IA and IB mandatory, e.g., not IIA and IB? The answer to this question will be given in a clear way in the discussion of the Hamiltonian viewpoint of the symmetries in Sec. VI.

\footnotetext{
${ }^{7}$ A real challenge would be to formulate the SF equation with an extended exchange symmetry that involves the three mass eigenstates, which is, however, far beyond the scope of this paper.
}

\section{F. Consistency check among the transformations}

It is important to verify the mutual consistency between the transformations that are involved in the symmetries. We do it here for symmetries IA- and IB-DMP, but it should be repeated when we find another new symmetry later.

The expressions of the eigenvalues $\lambda_{1}$ and $\lambda_{2}$ can be expressed as

$$
\begin{aligned}
& \lambda_{1} \equiv c_{\psi}^{2} \lambda_{-}+s_{\psi}^{2} \lambda_{0}-2 c_{\psi} s_{\psi} \mathcal{A}, \\
& \lambda_{2} \equiv s_{\psi}^{2} \lambda_{-}+c_{\psi}^{2} \lambda_{0}+2 c_{\psi} s_{\psi} \mathcal{A},
\end{aligned}
$$

where $\mathcal{A} \equiv \epsilon c_{12} s_{12} c_{\phi-\theta_{13}} \Delta m_{\text {ren }}^{2} \cdot{ }^{8}$ In symmetry IA-DMP, the transformation between $c_{\psi}$ and $s_{\psi}$ is such that $\mathcal{A}$ remains invariant. But since $c_{\psi} s_{\psi} \rightarrow-c_{\psi} s_{\psi}$, the eigenvalue exchange $\lambda_{1} \leftrightarrow \lambda_{2}$ occurs by the $\psi$ transformation. In symmetry IB-DMP, on the other hand, the transformation between $c_{\psi}$ and $s_{\psi}$ is such that $\mathcal{A}$ flips sign while $c_{\psi} s_{\psi}$ is invariant. Therefore, the transformations of $\lambda_{i}$ and $\psi$ are mutually consistent in both symmetries symmetry IA and symmetry IB in DMP.

\section{G. Symmetry IVB-DMP}

It would be a little cumbersome for the readers to follow the similar discussions for the remaining six symmetries. But we need to discuss at least one case in which the rephasing matrix $F$ matrix plays a role. Therefore, we investigate here the most involved case in which all the fundamental and dynamical parameters transform, which will be termed as "symmetry IVB-DMP." The results of the remaining five symmetries will be given in the summary Table I in Sec. V H, which also includes the ones discussed in the text.

We examine the case of class II and $\xi=\pi$. In class II, $\tau-\sigma=\xi \pm \pi=0(\bmod .2 \pi)$, there are only the two cases, $\tau=\sigma=0$ and $\tau=\sigma=\pi$. Since the former is already examined in Sec. V E which resulted in symmetry IB-DMP, we concentrate here on the case $\tau=\sigma=\pi$.

Within these restrictions, we can find the following two solutions: First solution: $\alpha=0, \beta=0$; and second solution: $\alpha=\pi, \beta=-\pi$. It is easy to recognize, by following the procedure described in Sec. VD, that the above two solutions lead to the solutions of the first condition (5.11) as

$$
c_{\psi^{\prime}}= \pm s_{\psi}, \quad s_{\psi^{\prime}}= \pm c_{\psi}
$$

where the upper (lower) sign in Eq. (5.17) corresponds to the first (second) solution of $\alpha, \beta$. Notice that due to the nonvanishing flavor basis neutrino rephasing phases, $\tau$ and $\sigma$, the vacuum mixing angles $\theta_{23}$ and $\theta_{13}$ must transform as

\footnotetext{
${ }^{8} \lambda_{-}$and $\lambda_{0}$ are the pre- $\theta_{12}$ rotation eigenvalues, which are identical to the ones in Ref. [24]. $\lambda_{-}$and $\lambda_{0}$ are the second-largest and smallest eigenvalues in the asymptotic region $a \rightarrow+\infty$ in the normal mass ordering.
} 
TABLE II. The case of $\tau=\sigma+\xi$ is called class I, and $\tau=\sigma+\xi \pm \pi$ as class II. The labels "upper" and "lower" imply the upper and lower signs in the corresponding columns in Table I.

\begin{tabular}{|c|c|c|}
\hline Symmetry & Class I or II, $\tau, \sigma, \xi$ & $\alpha, \beta$ \\
\hline Symmetry IA & Class I, $\tau=\sigma=0, \xi=0$ & $\begin{array}{c}\alpha=\beta=0 \text { (upper) } \\
\alpha=\pi, \beta=-\pi \text { (lower) }\end{array}$ \\
\hline Symmetry IB & Class II, $\tau=\sigma=0, \xi=\pi$ & $\begin{array}{c}\alpha=\pi, \beta=-\pi \text { (upper) } \\
\alpha=\beta=0 \text { (lower) }\end{array}$ \\
\hline Symmetry IIA & Class II, $\tau=0, \sigma=-\pi, \xi=0$ & $\begin{array}{l}\alpha=\pi, \beta=0 \text { (upper) } \\
\alpha=0, \beta=\pi \text { (lower) }\end{array}$ \\
\hline Symmetry IIB & Class I, $\tau=0, \sigma=-\pi, \xi=\pi$ & $\begin{array}{l}\alpha=0, \beta=\pi \text { (upper) } \\
\alpha=\pi, \beta=0 \text { (lower) }\end{array}$ \\
\hline Symmetry IIIA & Class II, $\tau=\pi, \sigma=0, \xi=0$ & $\begin{array}{l}\alpha=0, \beta=\pi \text { (upper) } \\
\alpha=\pi, \beta=0 \text { (lower) }\end{array}$ \\
\hline Symmetry IIIB & Class I, $\tau=\pi, \sigma=0, \xi=\pi$ & $\begin{array}{l}\alpha=\pi, \beta=0 \text { (upper) } \\
\alpha=0, \beta=\pi \text { (lower) }\end{array}$ \\
\hline Symmetry IVA & Class I, $\tau=\sigma=\pi, \xi=0$ & $\begin{array}{c}\alpha=\pi, \beta=-\pi \text { (upper) } \\
\alpha=\beta=0 \text { (lower) }\end{array}$ \\
\hline Symmetry IVB & Class II, $\tau=\sigma=\pi, \xi=\pi$ & $\begin{array}{c}\alpha=\beta=0 \text { (upper) } \\
\alpha=\pi, \beta=-\pi \text { (lower) }\end{array}$ \\
\hline
\end{tabular}

$\theta_{23} \rightarrow-\theta_{23}$ and $\theta_{13} \rightarrow-\theta_{13}$. Then, the $\theta_{13}$ transformation induces the $\phi \rightarrow-\phi$ transformation, because $\sin 2 \phi \propto$ $\sin 2 \theta_{13}$ [11]. Finally, the consistency with the second condition (5.10) requires the $\theta_{12}$ transform as $\theta_{12} \rightarrow-\theta_{12}$.

Thus, we have obtained a new symmetry that we call "symmetry IVB-DMP":

Symmetry IVB-DMP:

$\theta_{23} \rightarrow-\theta_{23}, \quad \theta_{13} \rightarrow-\theta_{13}, \quad \theta_{12} \rightarrow-\theta_{12}, \quad \delta \rightarrow \delta+\pi$,

$\lambda_{1} \leftrightarrow \lambda_{2}, \quad \phi \rightarrow-\phi, \quad c_{\psi} \rightarrow \pm s_{\psi}, \quad s_{\psi} \rightarrow \pm c_{\psi}$.

Consistency between the $\lambda_{1} \leftrightarrow \lambda_{2}$ exchange and the $\psi$ transformation is maintained. As in the case of symmetry IB-DMP, $\mathcal{A} \equiv \epsilon c_{12} s_{12} c_{\phi-\theta_{13}} \Delta m_{\text {ren }}^{2}$ flips sign while $c_{\psi} s_{\psi}$ is invariant. Thus, transformations of $\psi$ are consistent with the eigenvalue exchange. See Eq. (5.16). With regard to $\psi$, $s_{\psi} c_{\psi}=\frac{1}{2} \sin 2 \psi$ transform as [11]

$$
s_{\psi} c_{\psi}=\frac{\epsilon c_{12} s_{12} c_{\phi-\theta_{13}} \Delta m_{\mathrm{ren}}^{2}}{\lambda_{2}-\lambda_{1}} \rightarrow s_{\psi} c_{\psi}
$$

under the transformation in Eq. (5.18), and $\cos 2 \psi$ as

$$
c_{\psi}^{2}-s_{\psi}^{2}=\frac{\lambda_{0}-\lambda_{-}}{\lambda_{2}-\lambda_{1}} \rightarrow-\left(c_{\psi}^{2}-s_{\psi}^{2}\right)
$$

Therefore, the transformations of the vacuum variables, the fundamental parameters, and the dynamical variables are mutually fully consistent.

\section{H. The whole structure of $1 \leftrightarrow 2$ exchange symmetry in DMP}

We are ready to present all the solutions of the SF equation, thereby displaying the whole structure of $1 \leftrightarrow 2$ exchange symmetry in DMP. We do this by presenting Table I for the eight or the pair-doubled four symmetries in DMP. Interested readers can easily follow the procedure described in Sec. V D to reproduce these results. To help this task, we provide the supplementary information in Table II to show how each symmetry corresponds to which solution of the SF equation. As noticed before, symmetry IA-DMP in the first line in Tables I and II was noticed in Ref. [11], but the remaining seven symmetries are all new.

In Table I, the pairing of the type-A (no $\delta$ is involved) and type-B ( $\delta$ is involved) symmetries is clearly visible for all the symmetries I, II, III, and IV. It arises because, roughly speaking, the transformations $\theta_{12} \rightarrow-\theta_{12}$ and $\delta \rightarrow$ $\delta+\pi$ are equivalent for the symmetry purposes in one case, and simultaneous usage of both transformations produces a null transformation in the other, as their effects are both multiplicative. This structure is already implicit in the SOL convention of the $U$ matrix in which the factor $e^{ \pm i \delta}$ is attached to $s_{12} .{ }^{9}$ But, again, we emphasize that the symmetries are valid in the probabilities computed by using the $U$ matrix, e.g., in the PDG convention.

Can we claim that the symmetries of the $1 \leftrightarrow 2$ state exchange type discussed above are all the possible

\footnotetext{
${ }^{9}$ One should not misunderstand that the type-A and -B pairing of the symmetry is due to a trivial consequence of the $s_{12} e^{ \pm i \delta}$ structure. When the type-A symmetry becomes type-B, the $\psi$ transformation changes from $\sin 2 \psi \rightarrow-\sin 2 \psi$ to $\sin 2 \psi \rightarrow$ $\sin 2 \psi$ (symmetries I and IV) or vice versa (symmetries II and III). Thus, understanding the whole structure requires the SF equation.
} 
symmetries in DMP? Of course, we cannot because the ansatz we used to solve the SF equation are of limited ones. Nevertheless, in view of the remarkable completeness of the symmetry structure including the pairing of A- and B-type symmetries, the possibility may not be immediately excluded that the symmetries listed in Tables I and II exhaust all the possible $1 \leftrightarrow 2$ state exchange symmetries in DMP.

\section{HAMILTONIAN VIEW OF THE $1 \leftrightarrow 2$ EXCHANGE SYMMETRY IN MATTER}

We now discuss the Hamiltonian view of the reparametrization symmetry of the $1 \leftrightarrow 2$ state exchange type in matter. We primarily treat in this section the DMP system, because the symmetries in the ZS system and in the vacuum can easily be understood as one of the DMP's symmetries with appropriate replacements of the variables. It is also worthwhile to mention that our focus on these $1 \leftrightarrow 2$ exchange symmetries in DMP is due to their rich varieties, exhibiting interesting features.

\section{A. Hamiltonian view of the DMP system}

The flavor basis Hamiltonian can be written in two ways, by using the fundamental variables, which we call $H_{\text {LHS }}$, or by the dynamical (i.e., Hamiltonian diagonalizing) variables, which we call $H_{\mathrm{RHS}}$. Of course, they must be equal,

$$
H_{\mathrm{LHS}}=H_{\mathrm{RHS}} .
$$

The explicit expressions of $H_{\mathrm{LHS}}$, and $H_{\mathrm{RHS}}$ in the DMP decomposed form into the unperturbed and perturbed terms read in the SOL convention as

$$
\begin{aligned}
& 2 E H_{\mathrm{LHS}}=U_{23}\left(\theta_{23}\right) U_{13}\left(\theta_{13}\right) U_{12}\left(\theta_{12}, \delta\right)\left[\begin{array}{ccc}
m_{1}^{2} & 0 & 0 \\
0 & m_{2}^{2} & 0 \\
0 & 0 & m_{3}^{2}
\end{array}\right] U_{12}\left(\theta_{12}, \delta\right)^{\dagger} U_{13}\left(\theta_{13}\right)^{\dagger} U_{23}\left(\theta_{23}\right)^{\dagger}+\left[\begin{array}{ccc}
a(x) & 0 & 0 \\
0 & 0 & 0 \\
0 & 0 & 0
\end{array}\right] \text {, } \\
& 2 E H_{\mathrm{RHS}}=U_{23}\left(\theta_{23}\right) U_{13}(\phi) U_{12}(\psi, \delta)\left\{\left[\begin{array}{ccc}
\lambda_{1} & 0 & 0 \\
0 & \lambda_{2} & 0 \\
0 & 0 & \lambda_{3}
\end{array}\right]+\epsilon c_{12} s_{12} \sin \left(\phi-\theta_{13}\right) \Delta m_{\mathrm{ren}}^{2}\left[\begin{array}{ccc}
0 & 0 & -s_{\psi} \\
0 & 0 & c_{\psi} e^{-i \delta} \\
-s_{\psi} & c_{\psi} e^{i \delta} & 0
\end{array}\right]\right\} \\
& \times U_{12}^{\dagger}(\psi, \delta) U_{13}^{\dagger}(\phi) U_{23}^{\dagger}\left(\theta_{23}\right) \text {. }
\end{aligned}
$$

Notice that $H_{\mathrm{RHS}}$ is shown as the decomposed form, but it is exact, namely, no approximation is made to derive Eq. (6.3). We denote the first and second terms in $H_{\text {LHS }}$ in Eq. (6.2) as $H_{\text {vac }}$ and $H_{\text {matt }}$, respectively. Similarly, we denote the first and second terms in $H_{\mathrm{RHS}}$ in Eq. (6.3) as $H_{0}$ and $H_{1}$, respectively. That is, $H_{\mathrm{LHS}}=H_{\mathrm{vac}}+H_{\text {matt }}$ and $H_{\mathrm{RHS}}=H_{0}+H_{1}$.

\section{B. Transformation of $\boldsymbol{H}_{\mathrm{LHS}}$}

Let us first understand how $H_{\text {LHS }}$ in Eq. (6.2) transform. $H_{\text {vac }}$ transforms only by the fundamental (i.e., vacuum mixing) parameters, and $H_{\text {matt }}$ remains intact. For symmetry IA, obviously $H_{\text {vac }}$ does not transform, as no vacuum parameter transforms. For symmetry IB, the vacuum parameters change as $\theta_{12} \rightarrow-\theta_{12}$ and $\delta \rightarrow \delta+\pi$, but they keep $U_{\text {SOL }}$ and hence $H_{\text {vac }}$ invariant, as they come into the combination $s_{12} e^{ \pm i \delta}$. Therefore, $H_{\mathrm{LHS}}$ is invariant under both symmetries IA and IB.

For symmetry $\mathrm{X}$, for $\mathrm{X}=$ IIA and IIB (see Table I), one can easily show that $H_{\text {vac }}$ transforms the vacuum parameters transformation as
$H_{\mathrm{vac}} \equiv\left[\begin{array}{ccc}h_{e e} & h_{e \mu} & h_{e \tau} \\ h_{\mu e} & h_{\mu \mu} & h_{\mu \tau} \\ h_{\tau e} & h_{\tau \mu} & h_{\tau \tau}\end{array}\right] \rightarrow\left[\begin{array}{ccc}h_{e e} & -h_{e \mu} & h_{e \tau} \\ -h_{\mu e} & h_{\mu \mu} & -h_{\mu \tau} \\ h_{\tau e} & -h_{\tau \mu} & h_{\tau \tau}\end{array}\right]$,

leaving a lozenge position minus. Then, the transformation property of $H_{\mathrm{LHS}}=H_{\mathrm{vac}}+H_{\text {matt }}$ can be expressed as a phase redefinition, which is to be applied onto the flavor and mass eigenstates

$H_{\text {LHS }} \rightarrow \operatorname{Rep}(\mathrm{II}) H_{\mathrm{LHS}} \operatorname{Rep}(\mathrm{II})$ for $\mathrm{X}=\mathrm{IIA}$ and IIB,

where we have introduced the rephasing matrix Rep(II) as

$$
\operatorname{Rep}(\mathrm{II})=\left[\begin{array}{ccc}
-1 & 0 & 0 \\
0 & 1 & 0 \\
0 & 0 & -1
\end{array}\right]
$$

That is, $H_{\mathrm{LHS}}$ is invariant under the transformations of symmetries IIA and IIB up to the phase redefinition of the 
flavor and mass eigenstates. The similar exercise can be repeated for symmetry $\mathrm{X}, \mathrm{X}=$ IIIA-IIIB and IVA-IVB, with the results:

$$
\begin{aligned}
& H_{\mathrm{LHS}} \rightarrow \operatorname{Rep}(\mathrm{III}) H_{\mathrm{LHS}} \operatorname{Rep}(\mathrm{III}) \text { for } \mathrm{X}=\text { IIIA and IIIB, } \\
& H_{\mathrm{LHS}} \rightarrow \operatorname{Rep}(\mathrm{IV}) H_{\mathrm{LHS}} \operatorname{Rep}(\mathrm{IV}) \text { for } \mathrm{X}=\mathrm{IVA} \text { and IVB, }
\end{aligned}
$$

where we have similarly defined Rep(III) and Rep(IV) as

$$
\operatorname{Rep}(\mathrm{III})=\left[\begin{array}{ccc}
-1 & 0 & 0 \\
0 & 1 & 0 \\
0 & 0 & 1
\end{array}\right], \quad \operatorname{Rep}(\mathrm{IV})=\left[\begin{array}{ccc}
-1 & 0 & 0 \\
0 & -1 & 0 \\
0 & 0 & 1
\end{array}\right]
$$

Notice that all the rephasing matrix $\operatorname{Rep}(\mathrm{X})$ does not distinguish between the A- and B-type symmetries. Thus, the transformation property of $H_{\mathrm{LHS}}$ can be expressed as a phase redefinition, which is to be applied onto the flavor and mass eigenstates.

\section{Transformation of $\boldsymbol{H}_{\mathrm{RHS}}$}

We move on to the transformations of $H_{\mathrm{RHS}}$ under symmetry $\mathrm{X}$, where $\mathrm{X}=\mathrm{IA}$, IB, IIA, IIB, IIIA, IIIB,
IVA, and IVB. To make our treatment simpler we prepare some minimal formulas here. We denote that $U_{12}(\psi, \delta) \rightarrow$ $U_{12}(X)$ under the transformations of symmetry X. An explicit computation leads to the expressions

$$
\begin{gathered}
U_{12}(X)=\left[\begin{array}{ccc}
\mp s_{\psi} & \pm c_{\psi} e^{i \delta} & 0 \\
\mp c_{\psi} e^{-i \delta} & \mp s_{\psi} & 0 \\
0 & 0 & 1
\end{array}\right] \quad \text { for } \mathrm{X}=\mathrm{IA}, \mathrm{IVA}, \\
U_{12}(X)=\left[\begin{array}{ccc} 
\pm s_{\psi} & \pm c_{\psi} e^{i \delta} & 0 \\
\mp c_{\psi} e^{-i \delta} & \pm s_{\psi} & 0 \\
0 & 0 & 1
\end{array}\right] \quad \text { for } \mathrm{X}=\text { IIA, IIIA, }
\end{gathered}
$$

where the \pm signs are commensurate with those for the A-type symmetries in Table I. $U_{12}(X)$ of the B-type can be obtained from the A-type one by the sign flip, $\pm \rightarrow \mp$ and $\mp \rightarrow \pm$ in Eq. (6.9).

\section{Transformation of $\mathrm{H}_{0}$}

We discuss the transformation of $H_{0}$. One can show that

$$
\begin{aligned}
& U_{12}(\psi, \delta) \operatorname{diag}\left(\lambda_{1}, \lambda_{2}, \lambda_{3}\right) U_{12}(\psi, \delta)^{\dagger} \rightarrow U_{12}(X) \operatorname{diag}\left(\lambda_{2}, \lambda_{1}, \lambda_{3}\right) U_{12}(X)^{\dagger} \\
& =\operatorname{Rep}(\mathrm{X}) U_{12}(\psi, \delta) \operatorname{diag}\left(\lambda_{1}, \lambda_{2}, \lambda_{3}\right) U_{12}(\psi, \delta)^{\dagger} \operatorname{Rep}(\mathrm{X}) .
\end{aligned}
$$

It is important to know that the rephasing matrices $\operatorname{Rep}(\mathrm{X})$ 's for the transformation of $H_{\mathrm{RHS}}$ are the same as those of $H_{\mathrm{LHS}}$. For $\mathrm{X}=\mathrm{IA}$ and $\mathrm{IB}, \operatorname{Rep}(\mathrm{I})=1$, which means that the $U_{12}(\psi, \delta)$ part of $H_{0}$ is invariant under the transformation of $\mathrm{X}=\mathrm{IA}$ and IB. For the rest of X, X = II, III, and IV, Rep (X) are given in Eqs. (6.6) and (6.8) which imply that the $U_{12}(\psi, \delta)$ part of $H_{0}$ is invariant under the transformation up to the rephasing matrix $\operatorname{Rep}(\mathrm{X}) .{ }^{10}$ Notice again that $\operatorname{Rep}(\mathrm{X})$ is the same for the A- and B-types for all $\mathrm{X}$.

\section{Transformation of $\mathrm{H}_{1}$}

Similarly, the transformation of the $U_{12}(X)$ part of $H_{1}$ can be worked out to lead to

$$
\begin{aligned}
& \left.U_{12}(\psi, \delta)\left[\begin{array}{ccc}
0 & 0 & -s_{\psi} \\
0 & 0 & c_{\psi} e^{-i \delta} \\
-s_{\psi} & c_{\psi} e^{i \delta} & 0
\end{array}\right] U_{12}(\psi, \delta)^{\dagger} \rightarrow U_{12}(X)\left[\begin{array}{ccc}
0 & 0 & -s_{\psi} \\
0 & 0 & c_{\psi} e^{-i \delta} \\
-s_{\psi} & c_{\psi} e^{i \delta} & 0
\end{array}\right]\right|_{\mathrm{X} \text { transformed }} U_{12}(X)^{\dagger} \\
& =\operatorname{Sign}(\mathrm{X}) \operatorname{Rep}(\mathrm{X}) U_{12}(\psi, \delta)\left[\begin{array}{ccc}
0 & 0 & -s_{\psi} \\
0 & 0 & c_{\psi} e^{-i \delta} \\
-s_{\psi} & c_{\psi} e^{i \delta} & 0
\end{array}\right] U_{12}(\psi, \delta)^{\dagger} \operatorname{Rep}(\mathrm{X}),
\end{aligned}
$$

where the prescription $\left.\right|_{\mathrm{X} \text { transformed }}$ implies that the transformed variables of $\psi$ and $\delta$ by the $\mathrm{X}$ transformation are to be inserted, which, of course, depend on $\mathrm{X}$. $\operatorname{Rep}(\mathrm{X})$ is the same as defined in Eqs. (6.6) and (6.8). $\operatorname{Sign}(X)=+$ for IA, IIB, IIIA, IVB, and $\operatorname{Sign}(X)=-$ for IB, IIA, IIIB, IVA.

\footnotetext{
${ }^{10}$ As a matter of fact, Rep(IV) does nothing as far as the transformation property of $H_{0}$ is concerned for $\mathrm{X}=$ IVA and IVB. But, it is introduced to match the transformation property of $H_{\mathrm{LHS}}$.
} 
In order for $H_{1}$ to be invariant under these transformations, the minus sign from $\operatorname{Sign}(\mathrm{X})$ must be canceled by an extra minus sign supplied from the prefactor $\epsilon c_{12} s_{12} \sin \left(\phi-\theta_{13}\right) \Delta m_{\text {ren }}^{2}$ in Eq. (6.3). It is not difficult to show that it indeed occurs. For IB and IIA, flipping the sign of $\theta_{12}$ absorbs the minus sign. For IIIB and IVA flipping the sign of $\theta_{13}-\phi$ cancels the minus sign. For IA and IIB no sign change occurs in the prefactor, and for IIIA and IVB the sign change occurs twice, by flipping $\theta_{12}$ and $\theta_{13}-\phi$, resulting in no net change in sign. These features can easily be confirmed by consulting Table I.

So far we have discussed how the transformations affect the part $\mathcal{O}$ sandwiched by $U_{12}(X)$ and $U_{12}(X)^{\dagger}$ in $H_{\mathrm{RHS}}$. The rephasing matrix $\operatorname{Rep}(X)$ that appeared in the transformed part as $\operatorname{Rep}(\mathrm{X}) U_{12}(X) \mathcal{O} U_{12}(X)^{\dagger} \operatorname{Rep}(\mathrm{X})$ must be extracted to both ends of the Hamiltonian. It is good to see that passing through the rephasing matrix remedies the flipped sign of $\sin \theta_{i j}$ for $i j=23,13$, and 12. Also one might wonder if passing through the rephasing matrix might affect the other rotation matrix when correcting the flipped sign of only $\sin \theta_{23}$ and $\sin \theta_{13}$ in symmetry II and symmetry III, respectively. Upon computation, one recognizes that this is carefully avoided.

Thus, we have shown that $H_{\mathrm{RHS}}$ is invariant up to the phase redefinitions of the flavor and mass eigenstates with the same rephasing matrix $\operatorname{Rep}(\mathrm{X})$ as used for $H_{\mathrm{LHS}}$ for each symmetry $\mathrm{X}$. The fact that the different rephasing matrices $\operatorname{Rep}(\mathrm{X})$ are necessary for $\mathrm{X}=\mathrm{I}$, II, III, and IV, but they are the same for the type-A and type-B cases in each Symmetry $X$, justifies the type-A and type-B pairing scheme given in Table I.

Finally, we note that essentially the same manipulation as used to show the invariance of $H_{0}$ in symmetries IA and IB can be used to show that the Hamiltonian of the ZS system as well as in the vacuum is invariant under symmetries IA and IB.

\section{Implications of symmetry as a Hamiltonian symmetry}

In conclusion of our treatment in the previous sections, we have shown that both $H_{\mathrm{LHS}}$ and $H_{\mathrm{RHS}}$ in the flavor basis Hamiltonians (6.2) and (6.3) are invariant under the transformations of the eight $1 \leftrightarrow 2$ exchange symmetries, symmetry IA, IB, ..., IVB given in Table I, up to the phase redefinitions of the flavor and mass eigenstates except for IA and IB. Therefore, these symmetries are the Hamiltonian symmetries. In Sec. VII, we will clarify the relationship between this statement and the similar one made in Ref. [12].

What is the implication of the symmetry being the Hamiltonian symmetry? This is one of the most important questions in this paper, and we can make the following statements:

(i) The Hamiltonian symmetry holds to all orders of perturbation theory. (ii) The symmetry is valid for the non-uniform matter density profile, as far as the adiabaticity condition holds, that is, under a mild variation of the matter density. ${ }^{11}$

\section{NATURE OF THE $1 \leftrightarrow 2$ EXCHANGE SYMMETRY IN NEUTRINO OSCILLATION IN MATTER}

Can we draw a unified picture of all the $1 \leftrightarrow 2$ exchange symmetries in matter we have uncovered so far? Symmetry IA-ZS, IB-ZS, and IA-DMP are the pure dynamical symmetries in the sense that the symmetry transformations are generated purely by the Hamiltonian diagonalizing, or the matter-dressed variables, and no fundamental variable [i.e., variable in the original Hamiltonian (6.2)] is involved. By the symmetry transformations $H_{\text {LHS }}$ in Eq. (6.2) is left intact, whereas $H_{\mathrm{RHS}}$ transforms. But, of course, $H_{\mathrm{RHS}}$ remains invariant without the rephasing matrix, as symmetry IA-ZS, IB-ZS, and IA-DMP are symmetries, as confirmed explicitly for the last one in Sec. VIC.

On the other hand, in symmetry IIA, IIB, IIIA, IIIB, IVA, IVB in DMP, $H_{\mathrm{LHS}}$ transforms by a part or all of the fundamental variables transformations. Even more interestingly $H_{\mathrm{RHS}}$ transforms by both the fundamental as well as the dynamical variables. As can be seen most clearly in symmetry IIA, IIB, IIIA, and IIIB in DMP, one cannot take a view that the transformations of the fundamental variables induce those of the dynamical variables. Of course, the transformations of the dynamical variables never generate the transformations of the fundamental variables, as is evident in symmetry IA-ZS, IB-ZS, and IA-DMP. Therefore, to summarize:

(i) In the reparametrization symmetry with the $1 \leftrightarrow 2$ state exchange in neutrino oscillation in matter, none of the fundamental and the dynamical variables that take part in the symmetry transformations are more basic. $^{12}$ They are just equally important and, of course, transform in a mutually consistent way with each other.

As mentioned in Sec. I, we have stated previously [12] about symmetry IA-DMP that "these symmetries as the dynamical symmetry, as opposed to the Hamiltonian symmetry." The statement refers to the feature that $H_{\mathrm{LHS}}$ in Eq. (6.2) does not transform by the symmetry IA. But, now under a more complete view with both $H_{\mathrm{LHS}}$ and $H_{\mathrm{RHS}}$ in our sight, we say that symmetry IA is also the

\footnotetext{
${ }^{11}$ We suspect that a Hamiltonian symmetry implies the symmetry in the oscillation probability for any matter density profile as far as it is nonsingular. While we look forward to investigating this point, for the time being, we try to remain in the safe side by imposing the adiabaticity condition.

${ }^{12}$ This characteristic may be reminiscent of "nuclear democracy" or "bootstrap," the phrases for a feature of the $S$ matrix theory to stress that none of the elementary or composite particles is fundamental [34].
} 
Hamiltonian symmetry, as $H_{\text {RHS }}$ receive the transformation under symmetry IA, albeit it is invariant, of course.

\section{CONCLUDING REMARKS}

In this paper we have discussed the $1 \leftrightarrow 2$ state relabeling, or reparametrization symmetry in neutrino oscillation in matter. We have developed a systematic method called the symmetry finder, for uncovering symmetry in the neutrino oscillation probability in matter. We first noticed that the Zaglauer-Schwarzer system possesses symmetries IA-ZS and IB-ZS (Sec. IV), the matter equivalent of the known symmetries in vacuum [14]. Then, we have applied the SF scheme to the Denton et al. [11] (DMP) framework formulated to first order in perturbation theory to uncover new symmetries of the $1 \leftrightarrow 2$ state exchange type. Thanks to this powerful method of the SF equation, we have identified the eight symmetries, symmetry IA, IB, IIA, IIB, IIIA, IIIB, IVA, IVB in DMP, which are summarized in Table I. They are all new symmetries apart from IA, which was found in Ref. [11] and has stimulated our interests toward this investigation.

We suspect that the above eight symmetries may be a complete set of the reparametrization symmetry of the $1 \leftrightarrow 2$ state exchange type, because of the special features of the above set of the symmetries, exhausting the possible ways of usage of the vacuum parameter transformation, as well as the A-type ( $\delta$ free) and B-type (with $\delta$ ) paired structure. But, since our ansatz used to solve the SF equation is a quite limited one, the above suspect cannot be a too strong one. Notwithstanding whether the suspect is true or not, to the best of our knowledge, we believe that this is the first systematic treatment of the symmetry hunting method in the neutrino oscillation probability in matter.

Probably, one of the nicest features in our treatment in this paper is ascribed to the demonstration of all the $1 \leftrightarrow 2$ state exchange reparametrization symmetry as the Hamiltonian symmetry. That is, the symmetry transformations make the flavor basis Hamiltonian invariant, possibly up to the neutrino flavor and mass eigenstate rephasing factors. Once a symmetry is elevated to a Hamiltonian symmetry, it follows that the symmetry holds to all orders in perturbation theory, even though it was originally found by using the first-order perturbation theory. It also follows that the Hamiltonian symmetry is valid for systems with varying matter densities.

We have restricted our discussions into the symmetry whose transformations keep the system into our world, thereby excluding the ones such as $\Delta m_{31}^{2} \rightarrow-\Delta m_{31}^{2}$, or $\theta_{12} \leq \frac{\pi}{4}$ to $\theta_{12} \geq \frac{\pi}{4}$. Then, our symmetry search has to be limited inherently to reparametrization or relabeling symmetries, apart from spacetime symmetries such as $C P T$ or $C P$. To our view this is an acceptable limitation. Then, one may ask what are more serious limitations that can or cannot be circumvented. In Sec. IV C, we have already discussed a difficulty in formulating the SF framework to include the $1 \leftrightarrow 3$ and $2 \leftrightarrow 3$ exchange symmetry. A real final challenge would be to formulate the SF equation with an extended exchange symmetry that involves the three mass eigenstates, which is, however, outside the scope of this paper.

One may ask: What is the utility of such symmetries? Our answer would be the following:

(i) The minimum practical utility of such symmetry is to serve for a consistency check of the calculated results of the oscillation probabilities.

(ii) Understanding the nature of the symmetry might produce interesting physical interpretations.

We note that the first merit is not so negligible if a researcher has to derive the expression of the oscillation probability in an isolated place, which could happen in pandemic eras.

However, the second point above may be more intriguing and appealing. Therefore, let us examine it a little further. An overview of the features of the symmetries discussed in this paper may be summarized as the mixed fundamental and dynamical symmetry, because the transformations are carried out partly by the fundamental (i.e., vacuum) and partly by the Hamiltonian-diagonalizing (matter-dressed) variables. It appears that none of them is more basic in nature for neutrino oscillations in matter, another example of "nuclear democracy."

But, then one may wonder why dynamical symmetry could arise from the system under the matter potential, but no neutrino self-interactions. Let us consider high-density neutrino gases in which neutrino-neutrino interactions play an important role. Such a fully interacting neutrino system would be the best place for an investigation of dynamical symmetry in its genuine sense. A mean-field treatment of such a system may result in the neutrino system with the external neutrino potential in its leading-order approximation. This system may be similar to the one we have discussed in this paper, apart from the difference between the external neutrino and electron potentials. If this reasoning is correct, we could have started seeing the dynamical variables and the symmetry associated with them. Hence, it would be very interesting to address the symmetry and mutual relationship between the fundamental and dynamical variables in such a system as the high density neutrino gases [35,36]. A particular aspect of the similar nonlinear system that is relevant for supernova neutrinos has been much discussed recently; see, e.g., Ref. [37] and the references therein.

\section{ACKNOWLEDGMENTS}

The author thanks Peter Denton and Stephen Parke for useful discussions and communications. The symmetry finder equation in vacuum, Eq. (3.2) in Ref. [14], has first been brought to his attention by Stephen Parke. 
[1] J. H. Christenson, J. W. Cronin, V. L. Fitch, and R. Turlay, Evidence for the $2 \pi$ Decay of the $K_{2}^{0}$ Meson, Phys. Rev. Lett. 13, 138 (1964).

[2] V. A. Naumov, Three neutrino oscillations in matter, $C P$ violation and topological phases, Int. J. Mod. Phys. D 01, 379 (1992).

[3] S. Toshev, On T violation in matter neutrino oscillations, Mod. Phys. Lett. A 06, 455 (1991).

[4] P. I. Krastev and S. T. Petcov, Resonance amplification and $T$-violation effects in three neutrino oscillations in the earth, Phys. Lett. B 205, 84 (1988).

[5] C. Jarlskog, Commutator of the Quark Mass Matrices in the Standard Electroweak Model and a Measure of Maximal CP Violation, Phys. Rev. Lett. 55, 1039 (1985).

[6] M. Kobayashi and T. Maskawa, $C P$ violation in the renormalizable theory of weak interaction, Prog. Theor. Phys. 49, 652 (1973).

[7] K. Kimura, A. Takamura, and H. Yokomakura, Exact formulas and simple $C P$ dependence of neutrino oscillation probabilities in matter with constant density, Phys. Rev. D 66, 073005 (2002).

[8] H. Minakata and H. Nunokawa, Exploring neutrino mixing with low-energy superbeams, J. High Energy Phys. 10 (2001) 001.

[9] H. Minakata, H. Nunokawa, and S. J. Parke, CP and T trajectory diagrams for a unified graphical representation of neutrino oscillations, Phys. Lett. B 537, 249 (2002).

[10] H. Nunokawa, S. J. Parke, and J. W. F. Valle, $C P$ violation and neutrino oscillations, Prog. Part. Nucl. Phys. 60, 338 (2008).

[11] P. B. Denton, H. Minakata, and S. J. Parke, Compact perturbative expressions for neutrino oscillations in matter, J. High Energy Phys. 06 (2016) 051.

[12] I. Martinez-Soler and H. Minakata, Perturbing neutrino oscillations around the solar resonance, Prog. Theor. Exp. Phys. 2019, 073 B07 (2019).

[13] H. Minakata, Neutrino amplitude decomposition in matter, Phys. Rev. D 103, 053004 (2021).

[14] S. Parke, Theoretical aspects of the quantum neutrino, arXiv: 1801.09643.

[15] H. W. Zaglauer and K. H. Schwarzer, The mixing angles in matter for three generations of neutrinos and the MSW mechanism, Z. Phys. C 40, 273 (1988).

[16] G. Barenboim, P. B. Denton, S. J. Parke, and C. A. Ternes, Neutrino oscillation probabilities through the looking glass, Phys. Lett. B 791, 351 (2019).

[17] H. Minakata and S. Uchinami, Parameter degeneracy in neutrino oscillation-Solution network and structural overview, J. High Energy Phys. 04 (2010) 111.

[18] G. L. Fogli and E. Lisi, Tests of three flavor mixing in long baseline neutrino oscillation experiments, Phys. Rev. D 54, 3667 (1996).

[19] Z. Maki, M. Nakagawa, and S. Sakata, Remarks on the unified model of elementary particles, Prog. Theor. Phys. 28, 870 (1962).
[20] L. Wolfenstein, Neutrino oscillations in matter, Phys. Rev. D 17, 2369 (1978).

[21] I. Martinez-Soler and H. Minakata, Standard versus nonstandard $C P$ phases in neutrino oscillation in matter with non-unitarity, Prog. Theor. Exp. Phys. 2020, 063B01 (2020).

[22] P. A. Zyla et al. (Particle Data Group), Review of particle physics, Prog. Theor. Exp. Phys. 2020, 083 C01 (2020).

[23] I. Martinez-Soler and H. Minakata, Physics of parameter correlations around the solar-scale enhancement in neutrino theory with unitarity violation, Prog. Theor. Exp. Phys. 2020, 113B01 (2020).

[24] H. Minakata and S. J. Parke, Simple and compact expressions for neutrino oscillation probabilities in matter, J. High Energy Phys. 01 (2016) 180.

[25] S. Zhou, Symmetric formulation of neutrino oscillations in matter and its intrinsic connection to renormalization-group equations, J. Phys. G 44, 044006 (2017).

[26] S. P. Mikheyev and A. Y. Smirnov, Resonance amplification of oscillations in matter and spectroscopy of solar neutrinos, Yad. Fiz. 42, 1441 (1985) [Sov. J. Nucl. Phys. 42, 913 (1985)].

[27] B. T. Cleveland, T. Daily, R. Davis, Jr., J. R. Distel, K. Lande, C. K. Lee, P. S. Wildenhain, and J. Ullman, Measurement of the solar electron neutrino flux with the Homestake chlorine detector, Astrophys. J. 496, 505 (1998).

[28] A. de Gouvea, A. Friedland, and H. Murayama, The dark side of the solar neutrino parameter space, Phys. Lett. B 490 , 125 (2000).

[29] G. L. Fogli, E. Lisi, and A. Palazzo, Quasi energy independent solar neutrino transitions, Phys. Rev. D 65, 073019 (2002).

[30] V. D. Barger, K. Whisnant, S. Pakvasa, and R. J. N. Phillips, Matter effects on three-neutrino oscillations, Phys. Rev. D 22, 2718 (1980).

[31] P. B. Denton and R. Pestes, The impact of different parameterizations on the interpretation of $C P$ violation in neutrino oscillations, J. High Energy Phys. 05 (2021) 139.

[32] M. Blennow and A. Y. Smirnov, Neutrino propagation in matter, Adv. High Energy Phys. 2013, 972485 (2013).

[33] H. Minakata and H. Nunokawa, $C P$ violation versus matter effect in long baseline neutrino oscillation experiments, Phys. Rev. D 57, 4403 (1998).

[34] G. F. Chew, The Analytic S Matrix: A basis for Nuclear Democracy (W. A. Benjamin, Inc, New York, 1966).

[35] J. T. Pantaleone, Neutrino oscillations at high densities, Phys. Lett. B 287, 128 (1992).

[36] S. Samuel, Neutrino oscillations in dense neutrino gases, Phys. Rev. D 48, 1462 (1993).

[37] S. Hannestad, G. G. Raffelt, G. Sigl, and Y. Y. Y. Wong, Self-induced conversion in dense neutrino gases: Pendulum in flavour space, Phys. Rev. D 74, 105010 (2006); Erratum, Phys. Rev. D 76, 029901 (2007). 